\title{
Aristarco revisitado
}

\author{
Aristarchus revisited
}

\author{
T.B. de Oliveira, V.T. Lima, A.C. Bertuola* \\ Instituto Federal de Educação, Ciência e Tecnologia de São Paulo, São Paulo, SP, Brasil
}

Recebido em 23 de setembro de 2015. Aceito em 8 de novembro de 2015

\begin{abstract}
Usando as triangulações propostas pelo antigo astrônomo Aristarco, obtivemos neste trabalho o tempo total e a duração do máximo dos eclipses solares e calculamos o tempo total dos eclipses lunares. Para isso, usamos os valores do período orbital da Lua e os valores das razões entre as distâncias Terra-Lua e o diâmetro da Terra. Combinamos esses valores com outros dois parâmetros geométricos, conseguidos a partir do tratamento das imagens dos eclipses do Sol e da Lua. Os resultados foram comparados com valores atuais.
\end{abstract}

Palavras-chave: Aristarco, eclipse, anel de fogo.

Using triangulations proposed by the ancient astronomer Aristarchus, we obtained in this work the total time to lunar and solar eclipses and the maximum duration of solar eclipses. For this purpose, we used the values of the orbital period of the Moon and the values of the ratio between the Earth-Moon distance and the Earth's diameter. We combined them with two other geometric parameters obtained from the processing of images of the Sun and Moon eclipses. The results were compared with current values.

Keywords: Aristarchus, eclipse, ring of fire.

\section{Introdução}

Revisitar os trabalhos de Aristarco ( $\sim 300 \mathrm{aC})$ é realizar um estudo das características geométricas do Sol, da Lua, da Terra e das configurações entre eles, em especial os eclipses lunar e solar [1-3]. Para resgatar as ideias e os trabalhos deste cientista antigo, adotaremos um ponto de vista atual, na qual conhecemos com grande precisão o valor do diâmetro da Terra $\left.\right|^{1}$ as distâncias Terra-Lua e o período de revolução da Lua em torno da Terra [4]. Seus valores numéricos se encontram organizados na Tabela 1.

$\mathrm{Na}$ primeira razão da Tabela $1, d_{T L}^{P}$ se refere aquela distância onde a Lua está no perigeu (periélio), ou seja, na posição mais próxima da Terra. Na segunda razão, $d_{T L}$ é a distância média entre a Terra e Lua e na terceira, $d_{T L}^{A}$ é a distância relativa ao apogeu (afélio), que é a posição mais afastada da Lua na sua órbita. O denominador $\left(D_{T}\right)$ destas

*Endereço de correspondência: acbertuola@gmail.com.

${ }^{1} \mathrm{O}$ diâmetro da Terra foi estimado por Eratóstenes $(\sim 225 \mathrm{aC})$ com razoável precisão. O valor utilizado neste trabalho é $D_{T}=12740 \mathrm{~km}$.
Tabela 1: Grandezas astronômicas a respeito da Lua.

\begin{tabular}{ccccc}
\hline \multicolumn{3}{c}{ Razões } & & \multicolumn{2}{c}{ Período da Lua (dias) } \\
\hline$\frac{d_{T L}^{P}}{D_{T}}$ & $\frac{d_{T L}}{D_{T}}$ & $\frac{d_{T L}^{A}}{D_{T}}$ & Sideral & Sinódico \\
\hline 28 & 30 & 32 & 27,3 & 29,53 \\
\hline
\end{tabular}

divisões é o valor do diâmetro equatorial da Terra [5]. A aquisição das medidas atuais da distância TerraLua é realizada pela emissão de um feixe de laser, de um ponto na superfície da Terra, que é refletido na Lua e captado novamente na Terra, após um tempo cuidadosamente cronometrado. A precisão deste método é tão espantosa, que permitiu detectar o afastamento da Lua em relação à Terra, em aproximadamente três centímetros por ano. Provavelmente, Aristarco utilizou o período sinódico, pois é observado e medido desde a antiguidade. Neste trabalho, usaremos também o valor do período sideral. Seus valores serão usados e considerados exatos neste trabalho. O período da Lua em torno da Terra (sideral ou sinódico) é rotulado indiferentemente por 
Tabela 2: Tempos da fase anular e período orbital.

\begin{tabular}{lccc}
\hline Eclipse solar & Duração do máximo & Duração do eclipse & Magnitude \\
\hline Anular & 4,1 minutos & 2,5 horas & 0,96 \\
Total & 2,8 minutos & 1,2 horas & 1,05 \\
\hline
\end{tabular}

$T_{L}$, o tempo de duração máxima por ta e, usaremos te para a duração total do eclipse.

O eclipse 6 6 é um fenômeno que durante a história da humanidade causou muita admiração e curiosidade. Por despertar estas reações, vem sendo observado e registrado desde a antiguidade, tornando possível por meio destas datas vincular acontecimentos importantes na História. No caso do eclipse solar, o evento acontece quando a Lua se alinha entre o Sol e a Terra, sendo possível visualizar três tipos diferentes deste acontecimento: total, parcial e anular. Neste trabalho focaremos principalmente os eclipses solar anular e o solar total. ${ }^{2}$ cuja medição dos tempos de duração do máximd ${ }^{3}, 9,10$ e da duração total do eclipse 9 , 11] foram previstos, divulgados e reproduzidos aqui conforme a Tabela 2 .

O eclipse lunar acontece quando a Terra se posiciona entre o Sol e a Lua. Mentalmente uma imagem pode ser criada considerando os dois primeiros astros em repouso enquanto a Lua se movimenta no cone de sombra da Terra. Os valores do tempo de duração dos eclipses lunares [12], quando a Lua estiver próxima do afélio ou do periélid ${ }^{4}$ estão reunidos na Tabela 3.

Os dados das Tabelas 2 e 3 serão importantes para comparações futuras. No entanto, a maioria destes dados são apenas resultados de previsões e não são, realmente, os dados experimentais obtidos das observações do fenômeno.

Nas próximas seções vamos revisitar os trabalhos de Aristarco utilizando as imagens dos eclipses solar

Tabela 3: Tempos de duração dos eclipses lunares.

\begin{tabular}{lc}
\hline Lua & Tempo (horas) \\
\hline Afastada da Terra & 3,48 \\
Próxima da Terra & 3,32 \\
\hline
\end{tabular}

\footnotetext{
${ }^{2}$ Anular: 20 de outubro de 2005. Total: 20 de março de 2015. ${ }^{3}$ Para o eclipse anular visto da Terra, a duração do máximo é aquela em que se tem a existência do anel de fogo. Para o eclipse solar total visto da Terra, a duração do máximo é aquele tempo em que o Sol fica inteiramente oculto pela Lua. ${ }^{4}$ Data do apogeu da Lua: 01/04/2015. Data do eclipse lunar: 04/04/2015.

Data do perigeu da Lua: 06/10/2014. Data do eclipse: $08 / 10 / 2014$.
}

e lunar, combinados com os valores da Tabela 1 para calcular os tempos da fase tota $5^{5}$ de todos os eclipses e, a duração máxima para os eclipses solares.

Aristarco observou as fases da Lua e mediu os ângulos entre a Terra, a Lua e o Sol; observou os eclipses solar e lunar e mediu os tempos de ocorrência desses fenômenos. Combinou todos os dados experimentais recolhidos com algumas triangulações geométricas e obteve finalmente as distâncias absolutas da Terra à Lua e da Terra ao Sol. Seguindo um procedimento inverso ao de Aristarco, mostraremos em seguida, como estimar os tempos dos eclipses solares e lunares usando as distâncias absolutas da Terra-Lua e utilizando as imagens fotográficas dos eclipses lunar e solar.

\section{Aristarco, a Lua, o Sol e a Terra}

O primeiro trabalho de Aristarco considerado na ordem deste artigo, pode ser apresentado como um exercício proposto para os estudantes. Seu enunciado, tal como está registrado na Ref. [1] é reproduzido assim: "no século III a.C. o astrônomo grego Aristarco de Samos estimou a razão $\frac{d_{T S}}{d_{T L}}$ entre a distância da Terra ao Sol $\left(d_{T S}\right)$ e a distância da Terra à Lua $\left(d_{T L}\right)$, medindo o ângulo $\theta$ entre as direções em que a Lua e o Sol são vistos da Terra quando a Lua está exatamente meio cheia ${ }^{6}$ metade do disco lunar iluminado: veja a Fig. 1(a). O valor que ele obteve foi $\theta=87$. (a) Encontre a estimativa de Aristarco para $\frac{d_{T S}}{d_{T L}}$. (b) Com base nos valores atualmente conhecidos, $\frac{d_{T S}}{d_{T L}} \approx 389$, ache o valor real de $\theta$ e critique o método de Aristarco".

A Fig. 1(a) está na referência citada anteriormente [1], e também é apresentada em outras [2,3], as vezes com ligeiras diferenças. Aquele que se ocupar da resolução deste problema conforme a Fig. 1(a) e assim, exercitar seus conhecimentos de trigonometria, certamente chegará ao resultado

$$
\frac{d_{T S}}{d_{T L}}=\sec \theta
$$

\footnotetext{
${ }^{5}$ Tempo entre o primeiro e o último contato no eclipse. ${ }^{6}$ Quarto minguante ou quarto crescente.
} 
Os valores numéricos solicitados nos itens (a) e (b) são obtidos com a Eq. (1) e estão contidos na Tabela 3 .

Nota-se que a razão $\frac{d_{T S}}{d_{T L}}$ prevista por Aristarco é aproximadamente vinte vezes menor que o valor atual aceito, citado no item (b) da questão. No entanto, a diferença entre o ângulo obtido por Aristarco e o ângulo aceito atualmente é menor que três graus. Isso de fato requer um bom instrumento para medida de ângulo e mais ainda, requer uma observação muito apurada para identificar o instante correto, em que a Lua teria exatamente sua metade iluminada, já que entre uma observação e outra, se passa um dia e o instante exato procurado pode não ser identificado com acurácia. Também é possível notar na Tabela 4 que, uma pequena variação de minutos de ângulo implica em uma grande variação na razão. Desse ponto de vista, o erro cometido por Aristarco foi realmente muito grande, ou seja, o antigo e brilhante astrônomo poderia ter obtido uma boa aproximação, se cometesse um erro menor que minutos de ângulo, em relação ao ângulo atualmente aceito. A maior dificuldade na precisão das medidas é realmente de cunho tecnológico e experimental.

Uma observação derradeira dessa triangulação de Aristarco está na possibilidade do experimentador medir os ângulos, somente do ponto T' na superfície da Terra como foi mostrado na Fig. 1(b). Existe uma diferença entre esse ângulo experimental $\theta^{\exp }$, que é uma medida direta a partir do ponto $T^{\prime}$ e o ângulo teórico $\theta$ medido em relação ao centro da Terra. Após o uso das funções trigonométricas nos triângulos $S L T$ e $S L T$ ' da Fig. 1(b), eles podem ser

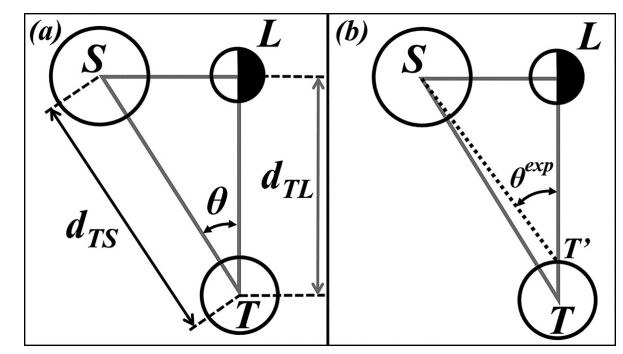

Figura 1: Triangulação proposta por Aristarco.

Tabela 4: Razão $\frac{d_{T S}}{d_{T L}}$ e o ângulo $\theta$ correspondente.

\begin{tabular}{ccccc}
\hline$\frac{d_{T S}}{d_{T L}}$ & 19,1 & 286,5 & 389,0 & 573,0 \\
$\theta$ & $87^{\circ}$ & $89^{\circ} 48^{\prime}$ & $89^{\circ} 51^{\prime} 10^{\prime \prime}$ & $89^{\circ} 54^{\prime}$ \\
\hline
\end{tabular}

relacionados entre si por

$$
\theta=\tan ^{-1}\left[\left(1-\frac{1}{2} \frac{D_{T}}{d_{T L}}\right) \tan \left(\theta^{\exp }\right)\right],
$$

em que o raio da Terra foi identificado pelo segmento $T T$ '. A razão $\frac{D_{T}}{d_{T L}}$ se mostra valiosa para estimar as discrepâncias geradas pela diferença entre os ângulos teórico e experimental. Por exemplo, usando o valor experimental $\theta^{\exp }=87^{\circ}$ obtido pelo astrônomo antigo e o valor médio da razão da Tabela 1 , o valor para o ângulo teórico correspondente obtido por meio da Eq. (2) vale $\theta=86^{\circ} 57^{\prime}$. Por isso, na abordagem deste trabalho, adotamos os ângulos $\theta^{\exp }$ e $\theta$ equivalentes.

Outro legado desse problema é perceber que, não adianta conhecer $d_{T L}$ com grande precisão, se o valores das razão (1) não estiverem bem avaliadas. Mas caso esta necessidade seja suprida, esta igualdade se mostra útil para calcular a distância absoluta Terra-Sol, com uma precisão razoável em relação a simplicidade adotada pelo astrônomo antigo.

\section{Fotografando o anel de fogo}

O método aqui desenvolvido passa pelo tratamento das imagens obtidas dos eclipses. Os eclipses, anular [13] e total 14 foram escolhidos como objetos de estudo e suas respectivas imagen 7 são mostradas na Fig. 2.

O eclipse anular se manifesta na fotografia quando o tamanho do Sol, que é representado pela região circular clara na Fig. 2, for menor que a região escura circular, que é o efeito visual da Lua vista da Terra encobrindo o Sol, mas deixando exposto um anel exterior visível como mostra a Fig. 2(a), denominado por anel de fogo. O eclipse total do Sol, mostrado na Fig. 2(b), ocorre quando seus raios são totalmente bloqueados pela presença da Lua. O eclipse parcial acontece quando apenas uma parte do Sol é

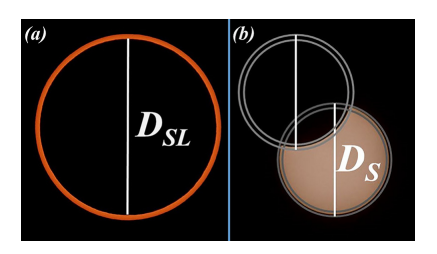

Figura 2: Eclipses total e anular do Sol.

\footnotetext{
${ }^{7}$ Créditos para:

(C) Stojan Stojanovsky

(C) 1999-2013 Luís Carreira - Pátio da Astronomia.
} 
encoberto pela Lua. Os diâmetros da região circular clara e da região circular escura na Fig. 2 são designados respectivamente por $D_{S}$ e $D_{S L}$. O quadro esquerdo nesta figura mostra o eclipse anular e o direito mostra o eclipse total. Nas regiões circulares são construídos dois círculos concêntricos 8 que englobam inteiramente a fronteira das regiões circulares clara e escura, conforme é mostrado na Fig. 2(b). Os círculos são construídos com o auxílio de um softwar $\mathrm{9}^{9}$ que contém o recurso de desenhar formas geométricas. Dois valores ${ }^{10}$ para cada diâmetro são obtidos com os adequados recursos do software, capaz de indicar as coordenadas cartesianas de um ponto na tela do computador. Esses valores contribuirão para estimar um erro para cada grandeza que será apresentada mais adiante nos resultados. Esta construção geométrica é repetida para as regiões claras e escuras nos quadros da Fig. 2 e assim, define-se de uma forma geral a razão

$$
\frac{D_{S L}}{D_{S}}=\alpha
$$

em que $\alpha$ é um dos parâmetros geométricos. Para este parâmetro, quatro valores são obtidos, para cada tipo de eclipse solar. Os resultados numéricos estão contidos na Tabela 5 .

As quantidades na Tabela 5 estão vinculados à habilidade do experimentador, seja nas construções geométricas ou nas suas coletas dos dados experimentais. Frequentemente foi necessário trabalhar com as imagens ampliadas, principalmente para determinar os pontos extremos para cada seguimento desenhado na Fig. 2, cuja distância entre eles representa o valor de um diâmetro.

Um esquema da triangulação do eclipse solar anular é exibido na Fig. 3.

Tabela 5: Valores de $\alpha$ para os eclipses anular e total.

\begin{tabular}{cccc}
\hline \multicolumn{3}{c}{ Parâmetro $\alpha$} \\
\hline \multicolumn{2}{c}{ anular } & \multicolumn{2}{c}{ total } \\
\hline 0,96 & 0,95 & 1,07 & 1,04 \\
0,95 & 0,93 & 1,03 & 0,99 \\
\hline
\end{tabular}

\footnotetext{
${ }^{8} \mathrm{Na}$ verdade esse procedimento não é obrigatório em absoluto. O leitor está livre para fazê-lo de outra maneira. O importante é obter experimentalmente os valores dos diâmetros das regiões circulares claras e escuras.

${ }^{9}$ Utilizamos as versões originais: o Power Point para desenhar e Photoshop para obter as coordenadas dos pontos.

${ }^{10}$ Para incluir a deformação na forma de cada astro, pode-se ajustar com elipses e então obter quatro valores para cada diâmetro.
}

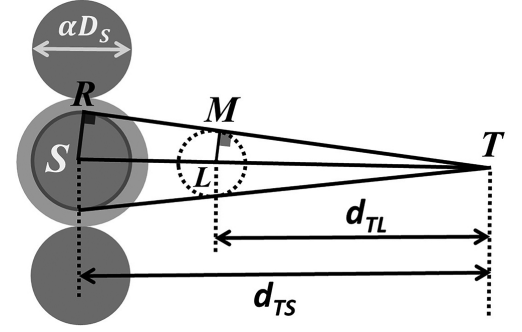

Figura 3: Geometria do eclipse anular do Sol.

O eclipse será anular se o valor do parâmetro $\alpha$ for menor que um, isto é, quando a posição da Lua estiver mais próxima do afélio. Para o eclipse total, o parâmetro geométrico assumirá valores igual ou maior que um, indicando que a Lua estará mais próxima do periélio. Usando a semelhança de triângulos $S R T$ e $L M T$ na Fig. 3, obtém-se a importante proporção

$$
\frac{d_{T S}}{D_{S}}=\alpha \frac{d_{T L}}{D_{L}} .
$$

Substituindo a Eq. (1) na Eq. (4), os diâmetros do Sol e da Lua ficam relacionados pela igualdade

$$
D_{L}=\frac{\alpha}{\sec \theta} D_{S}
$$

No intervalo de tempo $t_{a}$ no qual existe o anel de fogo ou durante o tempo total $t_{e}$ do eclipse, conjecturamos que o movimento da Lua é circular uniforme ${ }^{11}$ durante o eclipse. Para o eclipse anular, o raio da circunferência é a distância Terra-Lua, quando a Lua está no apogeu. No eclipse total do Sol, a trajetória circular tem um raio que é a distância Terra-Lua, quando a Lua estiver no perigeu. A imagem da Lua percorre uma distância $(1-\alpha) D_{S}$ enquanto existir o anel de fogo e a distância $(1+\alpha) D_{S}$, no tempo total de ocorrência do eclipse, conforme mostra a Fig. 3. Desse simples modelo físico, ou seja $\Delta s=\omega_{L} d_{T S} \Delta t$, com $\omega_{L}=\frac{2 \pi}{T_{L}}$, obtém-se

$$
\frac{d_{T S}}{D_{S}}=\frac{1-\alpha}{\delta_{a}}
$$

\footnotetext{
${ }^{11}$ Neste trabalho vamos considerar a aproximação circular para a trajetória da Lua durante o eclipse, seguindo a tradição dos gregos antigos. No entanto sabe-se de uma das leis de Kepler que a trajetória da Lua em torno do Sol é uma elipse. Usando a conservação do momento angular obtém-se a igualdade $\frac{\omega_{P}}{\omega_{A}}=$ $\left(\frac{d_{T L}^{A}}{d_{T L}^{P}}\right)^{2}$, que permite estimar os erros para as aproximações $\omega_{P}=\omega_{A} \simeq \frac{2 \pi}{T_{L}}$ usadas neste trabalho.
} 
após definir o valor do novo parâmetro físico por $\delta_{a}=\frac{2 \pi t_{a}}{T}$ e, de forma análoga,

$$
\frac{d_{T S}}{D_{S}}=\frac{1+\alpha}{\delta_{e}},
$$

em que $\delta_{e}=\frac{2 \pi t_{e}}{T}$. Com os resultados obtidos por meio das Eqs. (6) e (7), ambas razões na Eq. (4) ficam bem determinadas. No entanto, não é possível explicitar ainda os valores absolutos das grandezas astronômica: as distâncias Terra-Lua e Terra-Sol.

\section{O eclipse lunar na visão de Aristarco}

Para obter o valor absoluto da distância Terra-Lua $\left(d_{T L}\right)$, Aristarco buscou uma solução observando o eclipse lunar de duração máxima ${ }^{12}$ Hoje em dia é possível registrar esse fenômeno 15,16 com imagens, tal como a imagem mostrada na Fig. 4.

Para a determinação do diâmetro do cone de sombra $(D)$ em função do diâmetro da Lua, escolhe-se um ideal momento do vídeo em que a Lua está parcialmente dentro do cone de sombra, após o primeiro contato. Efetuando algumas construções geométricas na Fig. 4, da mesma maneira que foi feito na Fig. 2 é possível definir o segundo parâmetro geométrico

$$
\beta=\frac{D}{D_{L}} .
$$

Os valores numéricos para o parâmetro geométrico $\beta$ são obtidos para aqueles eclipses em que a Lua estará mais próxima do afélio ou do seu periélio. Esta escolha proposital é para se ter a chance de usar o parâmetro $\beta$ simultaneamente com o parâmetro $\alpha$.

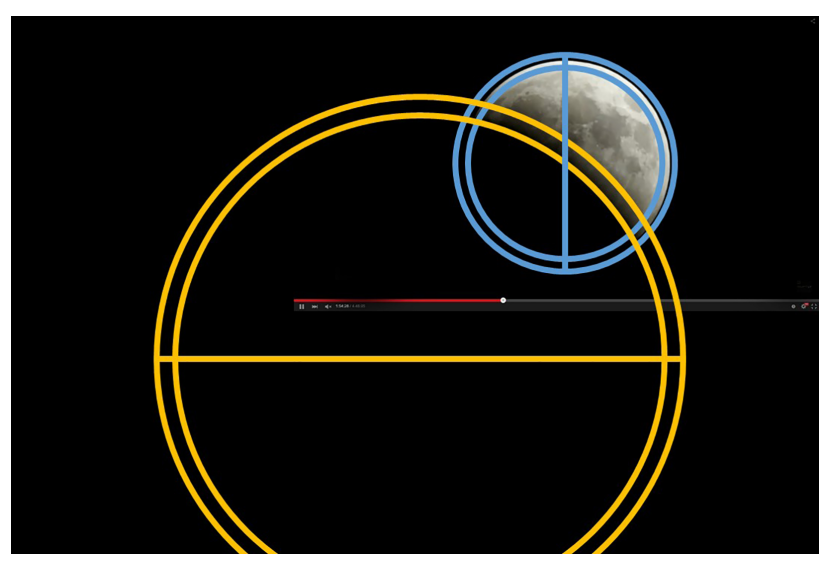

Figura 4: Construções geométricas no eclipse Lunar.

\footnotetext{
${ }^{12}$ A Lua percorre o diâmetro da secção circular do cone de sombra.
}

Isso acontecerá quando os parâmetros $(\alpha, \beta)$ corresponder as condições simultâneas (anular, afélio) ou (total, periélio). Os resultados numéricos do parâmetro $\beta$ que foram calculados conforme a descrição anterior estão contidos na Tabela 6 .

Outra triangulação [3] foi proposta pelo cientista em destaque neste artigo, cujo esquema geométrico é muito semelhante aquele mostrado na Fig. 5.

Na Fig. 5, a Lua pode ser identificada fora do cone de sombra, prestes a ser eclipsada. Sua trajetória pode ser aproximada por um segmento de reta de comprimento $D$. Identificando as semelhanças entre os triângulos $A_{0} S O, A_{2} T O$ e $A_{4} L O$ e utilizando as Eqs. (1), (3) e (8), obtém-se as seguintes proporções

$$
\frac{(1+\sec \theta) d_{T L}+x}{D_{S}}=\frac{d_{T L}+x}{D_{T}}=\frac{x}{\beta D_{L}},
$$

onde foram utilizadas as aproximações $A_{0} S \simeq D_{S}$ e $A_{2} T \simeq D_{T}$. Eliminando a incógnita $x$ na Eq. (9) obtém-se

$$
\frac{D_{L}}{D_{T}}=\frac{\gamma}{\beta(1+\gamma)},
$$

$\operatorname{com} \gamma=\frac{\alpha \beta(1+\sec \theta)}{\sec \theta-\alpha \beta}$, que é uma igualdade definida durante os cálculos. Combinando as Eqs. (4), (6) e (10), determina-se o valor absoluto da distância Terra-Lua em função do diâmetro da Terra por meio da equação

$$
\frac{d_{T L}}{D_{T}}=\frac{\gamma}{\alpha \beta \delta_{a, e}}\left(\frac{1 \mp \alpha}{1+\gamma}\right)
$$

\begin{tabular}{|c|c|c|c|}
\hline \multicolumn{4}{|c|}{ Parâmetro $\beta$} \\
\hline & & & élio \\
\hline 2,20 & 2,14 & 2,39 & 2,31 \\
\hline 2,14 & 2,08 & 2,22 & 2,14 \\
\hline
\end{tabular}

em que $\delta_{a, e}$ representa os dois parâmetros $\delta_{a}$ e $\delta_{e}$, de uma única maneira. A ordem dos índices é respectiva

Tabela 6: Valores de $\beta$ para o eclipse lunar.

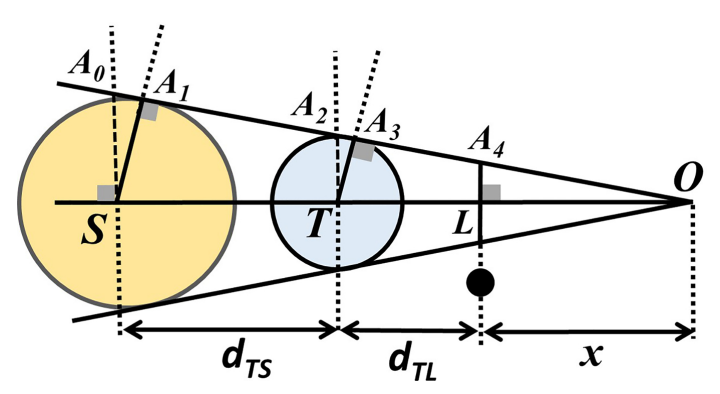

Figura 5: Esboço do eclipse Lunar. 
aos sinais negativo e positivo indicados no parêntese. Isolando os tempos na Eq. (11) obtém-se

$$
t_{e}=\frac{\gamma(1+\alpha) T_{L}}{2 \pi \alpha \beta(1+\gamma)}\left(\frac{d_{T S}}{D_{T}}\right)^{-1}
$$

ou então

$$
t_{a}=\frac{\gamma(1-\alpha) T_{L}}{2 \pi \alpha \beta(1+\gamma)}\left(\frac{d_{T L}}{D_{T}}\right)^{-1}
$$

que são os tempos referentes aos eclipses solares.

Admitindo também o movimento circular uniforme da Lua durante seu eclipse, tem-se

$$
D=\left(\frac{2 \pi}{T_{L}} d_{T L}\right) t_{L}
$$

O tempo de duração é calculado usando a igualdade (14) e as Eqs. (8) e (10). Após algumas manipulações matemáticas chega-se a equação

$$
t_{L}=\frac{\gamma(1+\beta) T_{L}}{2 \pi \beta(1+\gamma)}\left(\frac{d_{T L}}{D_{T}}\right)^{-1} .
$$

Os resultados obtidos com as Eqs. (12), 113) e (15) serão apresentados em seguida.

\section{Resultados obtidos}

Usando os dados da Tabela 5, os valores médios do parâmetro $\alpha$ são calculados e apresentados com seus respectivos erros, conforme é mostrado na Tabela 7 .

Comparando os valores na Tabela 7, com as magnitudes na Tabela 2, nota-se uma agradável concordância existente entre eles. Do ponto de vista prático, é possível aproveitar o erro estimado para o parâmetro $\alpha$, para selecionar as melhores imagens de um eclipse solar. Foi desta maneira que selecionamos as imagens do anel de fogo, usadas neste artigo. Analogamente elegem-se as melhores imagens de eclipses da Lua usando o erro estimado para o parâmetro $\beta$.

Os valores finais obtidos para os tempos nos eclipses solares estão organizados na Tabela 8.

Tabela 7: Valores para o parâmetro geométrico $\alpha$.

\begin{tabular}{lc}
\hline Eclipse & $\alpha$ \\
\hline Anular & $0,948 \pm 0,012$ \\
Total & $1,033 \pm 0,028$ \\
\hline
\end{tabular}

A Tabela 8 é uma divulgação dos resultados obtidos, da combinação entre um modelo físico matemático e os valores experimentais obtidos no tratamento de imagem. Todas os valores médios na Tabela 8 são quantidades típicas de tempos de duração de eclipses solares. Para o eclipse anular, os valores referentes ao período sinódico são aqueles mais próximos daqueles declarados na Tabela 2. Quanto ao eclipse total, o tempo de duração total do eclipse está superestimado, com seu valor mais próximos atribuído ao tempo sideral. Os valores da Tabela 8 concordam com outras previsões apresentadas na Tabela 2.

Os resultados para os tempos de duração dos eclipses lunares estão organizados na Tabela 9.

Para a Lua próxima do afélio, no eclipse lunar, os valores médios são muito próximos daqueles da Tabela 3 . Os valores obtidos e apresentados falam por si só e realmente mostram a força das triangulações propostas por Aristarco e a importância do caminho por ele adotado.

\section{Comentários finais}

Aristarco observou os tempos de duração dos eclipses solar e lunar, para determinar a distância absoluta entre a Terra e a Lua. Neste artigo, foram descritos os passos opostos aos seguidos por ele, ou seja, os valores dos tempos dos eclipses solares e lunares foram calculados, conhecendo-se a distância Terra-Lua a priori. Mesmo usando uma imagem de boa qualidade do eclipse lunar, houve uma dificuldade muito grande em ajustar as circunferências do cone de sombra, pois sua fronteira, destacada pela imagem da Lua na Fig. 4, contém uma nitidez natural muito duvidosa. Depois de todas aquelas

Tabela 8: Valores obtidos para os tempos dos eclipses solares.

\begin{tabular}{llcc}
\hline Eclipse & \multicolumn{1}{c}{ Duração } & sideral & sinódico \\
\hline Anular & $t_{\mathrm{a}}$ (minutos) & $3,38 \pm 0,74$ & $3,66 \pm 0,80$ \\
& $t_{\mathrm{e}}$ (horas) & $2,10 \pm 0,04$ & $2,27 \pm 0,05$ \\
Total & $t_{a}$ (minutos) & $2,1 \pm 1,3$ & $2,2 \pm 1,4$ \\
& $t_{e}$ (horas) & $1,99 \pm 0,09$ & $2,15 \pm 0,10$ \\
\hline
\end{tabular}

Tabela 9: Valores obtidos para os tempos dos eclipses lunares.

\begin{tabular}{lccc}
\hline Eclipse lunar & Tempo & sideral & sinódico \\
\hline Afélio & $t_{\mathrm{L}}$ (horas) & $3,21 \pm 0,03$ & $3,47 \pm 0,03$ \\
Periélio & $t_{\mathrm{L}}$ (horas) & $3,30 \pm 0,07$ & $3,56 \pm 0,07$ \\
\hline
\end{tabular}


aproximações adotadas ao longo dos cálculos, das deficiências nas imagens, enfim, considerando tudo isto, foi surpreendente a qualidade dos resultados obtidos, quando comparados com outras previsões. Por isso, consideramos este trabalho válido, do ponto de vista investigativo, histórico e pedagógico.

\section{Agradecimentos}

T.B. de Oliveira e V.T. Lima agradecem o CNPq pelo apoio financeiro. Os autores aproveitam esta oportunidade para realizarem um agradecimento especial para cada pessoa que contribuiu para o registro dos eclipses estudados neste artigo. Registramos aqui um agradecimento especial para o árbitro por suas valiosas observações.

\section{Referências}

[1] H.M. Nussenzveig, Curso de Física Básica, Volume 1 (Editora Edgard Blücher Ltda, São Paulo, 2002).

[2] A.P. French, Newtonian Mechanics (Norton, New York, 1971).

[3] T.S. Kuhn, A Revolução Copernicana (Edições 70, lisboa, 1990).

[4] A.P. Tonel e G.F. Marranghello, Revista Brasileira de Ensino de Física 35, 2310 (2013).

[5] D. Halliday, Fisica, Volume 1 (LTC, Rio de Janeiro, 2012).

[6] L.B.F. Clouzet e L. Sodrê Jr, Revista Brasileira de Ensino de Física 2, 64 (1980).

[7] S.H.B. Livi, Caderno Catarinense de Ensino de Física 10, 261 (1993).

[8] W. Lopes, Caderno Brasileiro de Ensino de Física 31, 400 (2014).

[9] Universidade de São Paulo: http://www.cdcc.usp. $\mathrm{br} / \mathrm{cda} / \mathrm{ef}$ emerides/e-s-1999-2050.html, acessado em $01 / 8 / 2015$.

[10] http://oal.ul.pt/oobservatorio/vol11/n7/ pagina4.html, acessado em 01/8/2015.

[11] http://eclipse.gsfc.nasa.gov/solar.html acessado em 01/8/2015.

[12] http://eclipse.gsfc.nasa.gov/lunar.html acessado em 02/8/2015.

[13] Disponível no site http://www.astrosurf.com/ carreira/esa2005.html, acessado em 31/7/2015.

[14] http://www.space.com/ 28880-total-solar-eclipse-2015-photos-gallery. html, acessado em 03/8/2015.

[15] Disponível no site https://www.youtube.com/ watch?v=eG3ZRFagmrU, acessado em 30/7/2015.

[16] Disponível em https://www.youtube.com/watch? $\mathrm{v}=1 \mathrm{GNq}-\mathrm{TbTHkU}$, acessado em 30/7/2015. 